\title{
Learning Word Meta-Embeddings
}

\author{
Wenpeng Yin and Hinrich Schütze \\ Center for Information and Language Processing \\ LMU Munich, Germany \\ wenpeng@cis. lmu.de
}

\begin{abstract}
Word embeddings - distributed representations of words - in deep learning are beneficial for many tasks in NLP. However, different embedding sets vary greatly in quality and characteristics of the captured information. Instead of relying on a more advanced algorithm for embedding learning, this paper proposes an ensemble approach of combining different public embedding sets with the aim of learning metaembeddings. Experiments on word similarity and analogy tasks and on part-of-speech tagging show better performance of metaembeddings compared to individual embedding sets. One advantage of metaembeddings is the increased vocabulary coverage. We release our metaembeddings publicly at http:// cistern.cis.lmu.de/meta-emb.
\end{abstract}

\section{Introduction}

Recently, deep neural network (NN) models have achieved remarkable results in NLP (Collobert and Weston, 2008; Sutskever et al., 2014; Yin and Schütze, 2015). One reason for these results are word embeddings, compact distributed word representations learned in an unsupervised manner from large corpora (Bengio et al., 2003; Mnih and Hinton, 2009; Mikolov et al., 2013a; Pennington et al., 2014).

Some prior work has studied differences in performance of different embedding sets. For example, Chen et al. (2013) show that the embedding sets HLBL (Mnih and Hinton, 2009), SENNA (Collobert and Weston, 2008), Turian (Turian et al., 2010) and Huang (Huang et al., 2012) have great variance in quality and characteristics of the semantics captured. Hill et al. (2014; 2015a) show that embeddings learned by $\mathrm{NN}$ machine translation models can outperform three representative monolingual embedding sets: word2vec (Mikolov et al., 2013b), GloVe (Pennington et al., 2014) and CW (Collobert and Weston, 2008). Bansal et al. (2014) find that Brown clustering, SENNA, CW, Huang and word2vec yield significant gains for dependency parsing. Moreover, using these representations together achieved the best results, suggesting their complementarity. These prior studies motivate us to explore an ensemble approach. Each embedding set is trained by a different NN on a different corpus, hence can be treated as a distinct description of words. We want to leverage this diversity to learn better-performing word embeddings. Our expectation is that the ensemble contains more information than each component embedding set.

The ensemble approach has two benefits. First, enhancement of the representations: metaembeddings perform better than the individual embedding sets. Second, coverage: metaembeddings cover more words than the individual embedding sets. The first three ensemble methods we introduce are CONC, SVD and $1 \mathrm{TON}$ and they directly only have the benefit of enhancement. They learn metaembeddings on the overlapping vocabulary of the embedding sets. CONC concatenates the vectors of a word from the different embedding sets. SVD performs dimension reduction on this concatenation. 1TON assumes that a metaembedding for the word exists, e.g., it can be a randomly initialized vector in the beginning, and uses this metaembedding to predict representations of the word in the individual embedding sets by projections - the resulting fine-tuned metaembedding is expected to contain knowledge from all individual embedding sets.

To also address the objective of increased coverage of the vocabulary, we introduce $1 \mathrm{TON}^{+}$, 
a modification of $1 \mathrm{TON}$ that learns metaembeddings for all words in the vocabulary union in one step. Let an out-of-vocabulary (OOV) word $w$ of embedding set ES be a word that is not covered by ES (i.e., ES does not contain an embedding for $w) .{ }^{1} 1 \mathrm{TON}^{+}$first randomly initializes the embeddings for OOVs and the metaembeddings, then uses a prediction setup similar to $1 \mathrm{TON}$ to update metaembeddings as well as OOV embeddings. Thus, $1 \mathrm{TON}^{+}$simultaneously achieves two goals: learning metaembeddings and extending the vocabulary (for both metaembeddings and invidual embedding sets).

An alternative method that increases coverage is Mutuallearning. Mutuallearning learns the embedding for a word that is an OOV in embedding set from its embeddings in other embedding sets. We will use MutualLearning to increase coverage for CONC, SVD and $1 \mathrm{TON}$, so that these three methods (when used together with MutualLearning) have the advantages of both performance enhancement and increased coverage.

In summary, metaembeddings have two benefits compared to individual embedding sets: enhancement of performance and improved coverage of the vocabulary. Below, we demonstrate this experimentally for three tasks: word similarity, word analogy and POS tagging.

If we simply view metaembeddings as a way of coming up with better embeddings, then the alternative is to develop a single embedding learning algorithm that produces better embeddings. Some improvements proposed before have the disadvantage of increasing the training time of embedding learning substantially; e.g., the NNLM presented in (Bengio et al., 2003) is an order of magnitude less efficient than an algorithm like word2vec and, more generally, replacing a linear objective function with a nonlinear objective function increases training time. Similarly, fine-tuning the hyperparameters of the embedding learning algorithm is complex and time consuming. In terms of coverage, one might argue that we can retrain an existing algorithm like word2vec on a bigger corpus. However, that needs much longer training time than our simple ensemble approaches which achieve coverage as well as enhancement with less effort. In many cases, it is not possible to retrain

\footnotetext{
${ }^{1}$ We do not consider words in this paper that are not covered by any of the individual embedding sets. OOV refers to a word that is covered by a proper subset of ESs.
}

using a different algorithm because the corpus is not publicly available. But even if these obstacles could be overcome, it is unlikely that there ever will be a single "best" embedding learning algorithm. So the current situation of multiple embedding sets with different properties being available is likely to persist for the forseeable future. Metaembedding learning is a simple and efficient way of taking advantage of this diversity. As we will show below they combine several complementary embedding sets and the resulting metaembeddings are stronger than each individual set.

\section{Related Work}

Related work has focused on improving performance on specific tasks by using several embedding sets simultaneously. To our knowledge, there is no work that aims to learn generally useful metaembeddings from individual embedding sets.

Tsuboi (2014) incorporates word2vec and GloVe embeddings into a POS tagging system and finds that using these two embedding sets together is better than using them individually. Similarly, Turian et al. (2010) find that using Brown clusters, CW embeddings and HLBL embeddings for Name Entity Recognition and chunking tasks together gives better performance than using these representations individually.

Luo et al. (2014) adapt CBOW (Mikolov et al., 2013a) to train word embeddings on different datasets - a Wikipedia corpus, search clickthrough data and user query data - for web search ranking and for word similarity. They show that using these embeddings together gives stronger results than using them individually.

Both (Yin and Schütze, 2015) and (Zhang et al., 2016) try to incorporate multiple embedding sets into channels of convolutional neural network system for sentence classification tasks. The better performance also hints the complementarity of component embedding sets, however, such kind of incorporation brings large numbers of training parameters.

In sum, these papers show that using multiple embedding sets is beneficial. However, they either use embedding sets trained on the same corpus (Turian et al., 2010) or enhance embedding sets by more training data, not by innovative learning algorithms (Luo et al., 2014), or make the system architectures more complicated (Yin and 


\begin{tabular}{l|rrr} 
& Vocab Size & Dim & Training Data \\
\hline HLBL (Mnih and Hinton, 2009) & 246,122 & 100 & Reuters English newswire August 1996-August 1997 \\
Huang (Huang et al., 2012) & 100,232 & 50 & April 2010 snapshot of Wikipedia \\
Glove (Pennington et al., 2014) & $1,193,514$ & 300 & 42 billion tokens of web data, from Common Crawl \\
CW (Collobert and Weston, 2008) & 268,810 & 200 & Reuters English newswire August 1996-August 1997 \\
word2vec (Mikolov et al., 2013b) & 929,022 & 300 & About 100 billion tokens from Google News
\end{tabular}

Table 1: Embedding Sets (Dim: dimensionality of word embeddings).

Schütze, 2015; Zhang et al., 2016). In our work, we can leverage any publicly available embedding set learned by any learning algorithm. Our metaembeddings (i) do not require access to resources such as large computing infrastructures or proprietary corpora; (ii) are derived by fast and simple ensemble learning from existing embedding sets; and (iii) have much lower dimensionality than a simple concatentation, greatly reducing the number of parameters in any system that uses them.

An alternative to learning metaembeddings from embeddings is the MVLSA method that learns powerful embeddings directly from multiple data sources (Rastogi et al., 2015). Rastogi et al. (2015) combine a large number of data sources and also run two experiments on the embedding sets Glove and word2vec. In contrast, our focus is on metaembeddings, i.e., embeddings that are exclusively based on embeddings. The advantages of metaembeddings are that they outperform individual embeddings in our experiments, that few computational resources are needed, that no access to the original data is required and that embeddings learned by new powerful (including nonlinear) embedding learning algorithms in the future can be immediately taken advantage of without any changes being necessary to our basic framework. In future work, we hope to compare MVLSA and metaembeddings in effectiveness (Is using the original corpus better than using embeddings in some cases?) and efficiency (Is using SGD or SVD more efficient and in what circumstances?).

\section{Experimental Embedding Sets}

In this work, we use five released embedding sets. (i) HLBL. Hierarchical log-bilinear (Mnih and Hinton, 2009) embeddings released by Turian et al. (2010); ${ }^{2}$ 246,122 word embeddings, 100 dimensions; training corpus: RCV1 corpus (Reuters English newswire, August 1996 - August 1997).

\footnotetext{
${ }^{2}$ metaoptimize.com/projects/wordreprs
}

(ii) Huang. ${ }^{3}$ Huang et al. (2012) incorporate global context to deal with challenges raised by words with multiple meanings; 100,232 word embeddings, 50 dimensions; training corpus: April 2010 snapshot of Wikipedia. (iii) GloVe ${ }^{4}$ (Pennington et al., 2014). 1,193,514 word embeddings, 300 dimensions; training corpus: 42 billion tokens of web data, from Common Crawl. (iv) CW (Collobert and Weston, 2008). Released by Turian et al. (2010); $;^{5}$ 268,810 word embeddings, 200 dimensions; training corpus: same as HLBL. (v) word2vec (Mikolov et al., 2013b) CBOW; ${ }^{6}$ 929,022 word embeddings (we discard phrase embeddings), 300 dimensions; training corpus: Google News (about 100 billion words). Table 1 gives a summary of the five embedding sets.

The intersection of the five vocabularies has size 35,965 , the union has size 2,788,636.

\section{Ensemble Methods}

This section introduces the four ensemble methods: CONC, SVD, $1 \mathrm{TON}$ and $1 \mathrm{TON}^{+}$.

\subsection{CONC: Concatenation}

In CONC, the metaembedding of $w$ is the concatenation of five embeddings, one each from the five embedding sets. For GloVe, we perform L2 normalization for each dimension across the vocabulary as recommended by the GloVe authors. Then each embedding of each embedding set is L2-normalized. This ensures that each embedding set contributes equally (a value between -1 and 1) when we compute similarity via dot product.

We would like to make use of prior knowledge and give more weight to well performing embedding sets. In this work, we give GloVe and word2vec weight $i>1$ and weight 1 to the other three embedding sets. We use MC30 (Miller and Charles, 1991) as dev set, since all embedding sets fully cover it. We set $i=8$, the value in Figure 1

\footnotetext{
${ }^{3}$ ai.stanford.edu/ ehhuang

${ }^{4}$ nlp.stanford.edu/projects/glove

5 metaoptimize.com/projects/wordreprs

${ }^{6}$ code.google.com/p/Word2Vec
} 
where performance reaches a plateau. After L2 normalization, GloVe and word2vec embeddings are multiplied by $i$ and remaining embedding sets are left unchanged.

The dimensionality of CONC metaembeddings is $k=100+50+300+200+300=950$. We also tried equal weighting, but the results were much worse, hence we skip reporting it. It nevertheless gives us insight that simple concatenation, without studying the difference among embedding sets, is unlikely to achieve enhancement. The main disadvantage of simple concatenation is that word embeddings are commonly used to initialize words in DNN systems; thus, the high-dimensionality of concatenated embeddings causes a great increase in training parameters.

\subsection{SVD: Singular Value Decomposition}

We do SVD on above weighted concatenation vectors of dimension $k=950$.

Given a set of CONC representations for $n$ words, each of dimensionality $k$, we compute an SVD decomposition $C=U S V^{T}$ of the corresponding $n \times k$ matrix $C$. We then use $U_{d}$, the first $d$ dimensions of $U$, as the SVD metaembeddings of the $n$ words. We apply L2-normalization to embeddings; similarities of SVD vectors are computed as dot products.

$d$ denotes the dimensionality of metaembeddings in SVD, $1 \mathrm{TON}$ and $1 \mathrm{TON}^{+}$. We use $d=$ 200 throughout and investigate the impact of $d$ below.

\subsection{TON}

Figure 2 depicts the simple neural network we employ to learn metaembeddings in 1 TON. White

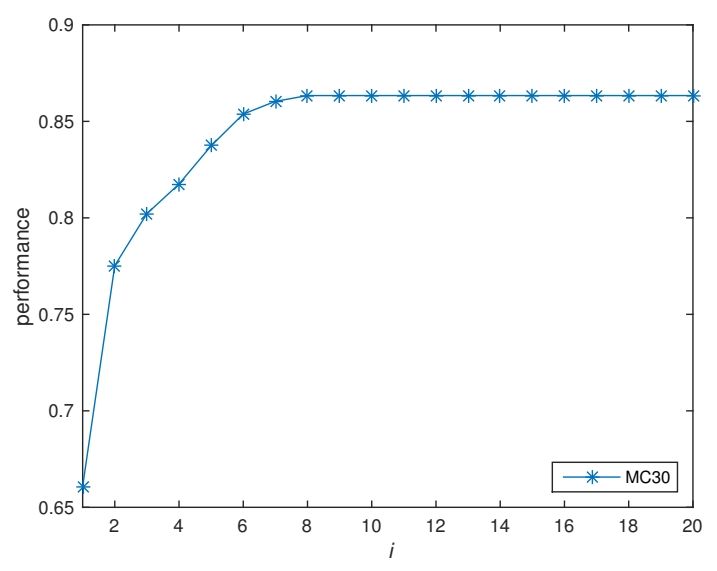

Figure 1: Performance vs. Weight scalar $i$ rectangles denote known embeddings. The target to learn is the metaembedding (shown as shaded rectangle). Metaembeddings are initialized randomly.

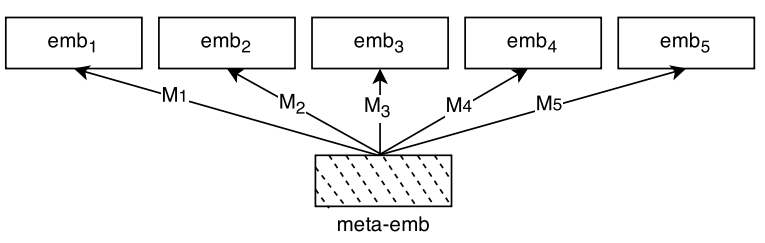

Figure 2: 1toN

Let $c$ be the number of embedding sets under consideration, $V_{1}, V_{2}, \ldots, V_{i}, \ldots, V_{c}$ their vocabularies and $V^{\cap}=\cap_{i=1}^{c} V_{i}$ the intersection, used as training set. Let $V_{*}$ denote the metaembedding space. We define a projection $f_{* i}$ from space $V_{*}$ to space $V_{i}(i=1,2, \ldots, c)$ as follows:

$$
\hat{\mathbf{w}}_{i}=\mathbf{M}_{* i} \mathbf{w}_{*}
$$

where $\mathbf{M}_{* i} \in \mathbb{R}^{d_{i} \times d}, \mathbf{w}_{*} \in \mathbb{R}^{d}$ is the metaembedding of word $w$ in space $V_{*}$ and $\hat{\mathbf{w}}_{i} \in \mathbb{R}^{d_{i}}$ is the projected (or learned) representation of word $w$ in space $V_{i}$. The training objective is as follows:

$$
E=\sum_{i=1}^{c} k_{i} \cdot\left(\sum_{\mathbf{w} \in V^{\cap}}\left|\hat{\mathbf{w}}_{i}-\mathbf{w}_{i}\right|^{2}+l_{2} \cdot\left|\mathbf{M}_{* i}\right|^{2}\right)
$$

In Equation 2, $k_{i}$ is the weight scalar of the $i^{t h} \mathrm{em}$ bedding set, determined in Section 4.1, i.e, $k_{i}=8$ for GloVe and word2vec embedding sets, otherwise $k_{i}=1 ; l_{2}$ is the weight of $\mathrm{L} 2$ normalization.

The principle of $1 \mathrm{TON}$ is that we treat each individual embedding as a projection of the metaembedding, similar to principal component analysis. An embedding is a description of the word based on the corpus and the model that were used to create it. The metaembedding tries to recover a more comprehensive description of the word when it is trained to predict the individual descriptions.

$1 \mathrm{TON}$ can also be understood as a sentence modeling process, similar to DBOW (Le and Mikolov, 2014). The embedding of each word in a sentence $s$ is a partial description of $s$. DBOW combines all partial descriptions to form a comprehensive description of $s$. DBOW initializes the sentence representation randomly, then uses this representation to predict the representations of individual words. The sentence representation of $s$ corresponds to the metaembedding in $1 \mathrm{TON}$; and the representations of the words in $s$ correspond to the five embeddings for a word in $1 \mathrm{TON}$. 


\section{$4.4 \mathrm{1toN}^{+}$}

Recall that an OOV (with respect to embedding set ES) is defined as a word unknown in ES. $1 \mathrm{TON}^{+}$ is an extension of $1 \mathrm{TON}$ that learns embeddings for OOVs; thus, it does not have the limitation that it can only be run on overlapping vocabulary.

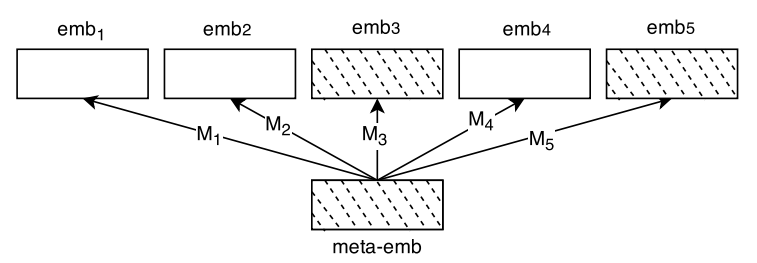

Figure 3: 1 toN ${ }^{+}$

Figure 3 depicts $1 \mathrm{TON}^{+}$. In contrast to Figure 2 , we assume that the current word is an OOV in embedding sets 3 and 5. Hence, in the new learning task, embeddings 1, 2, 4 are known, and embeddings 3 and 5 and the metaembedding are targets to learn.

We initialize all OOV representations and metaembeddings randomly and use the same mapping formula as for $1 \mathrm{TON}$ to connect a metaembedding with the individual embeddings. Both metaembedding and initialized OOV embeddings are updated during training.

Each embedding set contains information about only a part of the overall vocabulary. However, it can predict what the remaining part should look like by comparing words it knows with the information other embedding sets provide about these words. Thus, $1 \mathrm{TON}^{+}$learns a model of the dependencies between the individual embedding sets and can use these dependencies to infer what the embedding of an OOV should look like.

CONC, SVD and 1TON compute metaembeddings only for the intersection vocabulary. $1 \mathrm{TON}^{+}$ computes metaembeddings for the union of all individual vocabularies, thus greatly increasing the coverage of individual embedding sets.

\section{Mutuallearning}

MutualLearning is a method that extends CONC, SVD and 1TON such that they have increased coverage of the vocabulary. With MUTUALLEARNING, all four ensemble methods CONC, SVD, 1 TON and $1 \mathrm{TON}^{+}-$have the benefits of both performance enhancement and increased coverage and we can use criteria like performance, compactness and efficiency of training

\begin{tabular}{l|rll} 
& \multicolumn{1}{|c}{ bs } & \multicolumn{1}{c}{ lr } & \multicolumn{1}{c}{$l_{2}$} \\
\hline $1 \mathrm{TON}$ & 200 & 0.005 & $5 \times 10^{-4}$ \\
MUTUALLEARNING (ml) & 200 & 0.01 & $5 \times 10^{-8}$ \\
$1 \mathrm{TON}^{+}$ & 2000 & 0.005 & $5 \times 10^{-4}$
\end{tabular}

Table 2: Hyperparameters. bs: batch size; lr: learning rate; $l_{2}$ : L2 weight.

to select the best ensemble method for a particular application.

Mutuallearning is applied to learn OOV embeddings for all $c$ embedding sets; however, for ease of exposition, let us assume we want to compute embeddings for OOVs for embedding set $j$ only, based on known embeddings in the other $c-1$ embedding sets, with indexes $i \in\{1 \ldots j-$ $1, j+1 \ldots c\}$. We do this by learning $c-1$ mappings $f_{i j}$, each a projection from embedding set $E_{i}$ to embedding set $E_{j}$.

Similar to Section 4.3 , we train mapping $f_{i j}$ on the intersection $V_{i} \cap V_{j}$ of the vocabularies covered by the two embedding sets. Formally, $\hat{\mathbf{w}}_{j}=f_{i j}\left(\mathbf{w}_{i}\right)=\mathbf{M}_{i j} \mathbf{w}_{i}$ where $\mathbf{M}_{i j} \in \mathbb{R}^{d_{j} \times d_{i}}$, $\mathbf{w}_{i} \in \mathbb{R}^{d_{i}}$ denotes the representation of word $w$ in space $V_{i}$ and $\hat{\mathbf{w}}_{j}$ is the projected metaembedding of word $w$ in space $V_{j}$. Training loss has the same form as Equation 2 except that there is no " $\sum_{i=1}^{c} k_{i}$ " term. A total of $c-1$ projections $f_{i j}$ are trained to learn OOV embeddings for embedding set $j$.

Let $w$ be a word unknown in the vocabulary $V_{j}$ of embedding set $j$, but known in $V_{1}, V_{2}, \ldots, V_{k}$. To compute an embedding for $w$ in $V_{j}$, we first compute the $k$ projections $f_{1 j}\left(\mathbf{w}_{1}\right), f_{2 j}\left(\mathbf{w}_{2}\right), \ldots$, $f_{k j}\left(\mathbf{w}_{k}\right)$ from the source spaces $V_{1}, V_{2}, \ldots, V_{k}$ to the target space $V_{j}$. Then, the element-wise average of $f_{1 j}\left(\mathbf{w}_{1}\right), f_{2 j}\left(\mathbf{w}_{2}\right), \ldots, f_{k j}\left(\mathbf{w}_{k}\right)$ is treated as the representation of $w$ in $V_{j}$. Our motivation is that - assuming there is a true representation of $w$ in $V_{j}$ and assuming the projections were learned well - we would expect all the projected vectors to be close to the true representation. Also, each source space contributes potentially complementary information. Hence averaging them is a balance of knowledge from all source spaces.

\section{Experiments}

We train NNs by back-propagation with AdaGrad (Duchi et al., 2011) and mini-batches. Table 2 gives hyperparameters.

We report results on three tasks: word similarity, word analogy and POS tagging. 


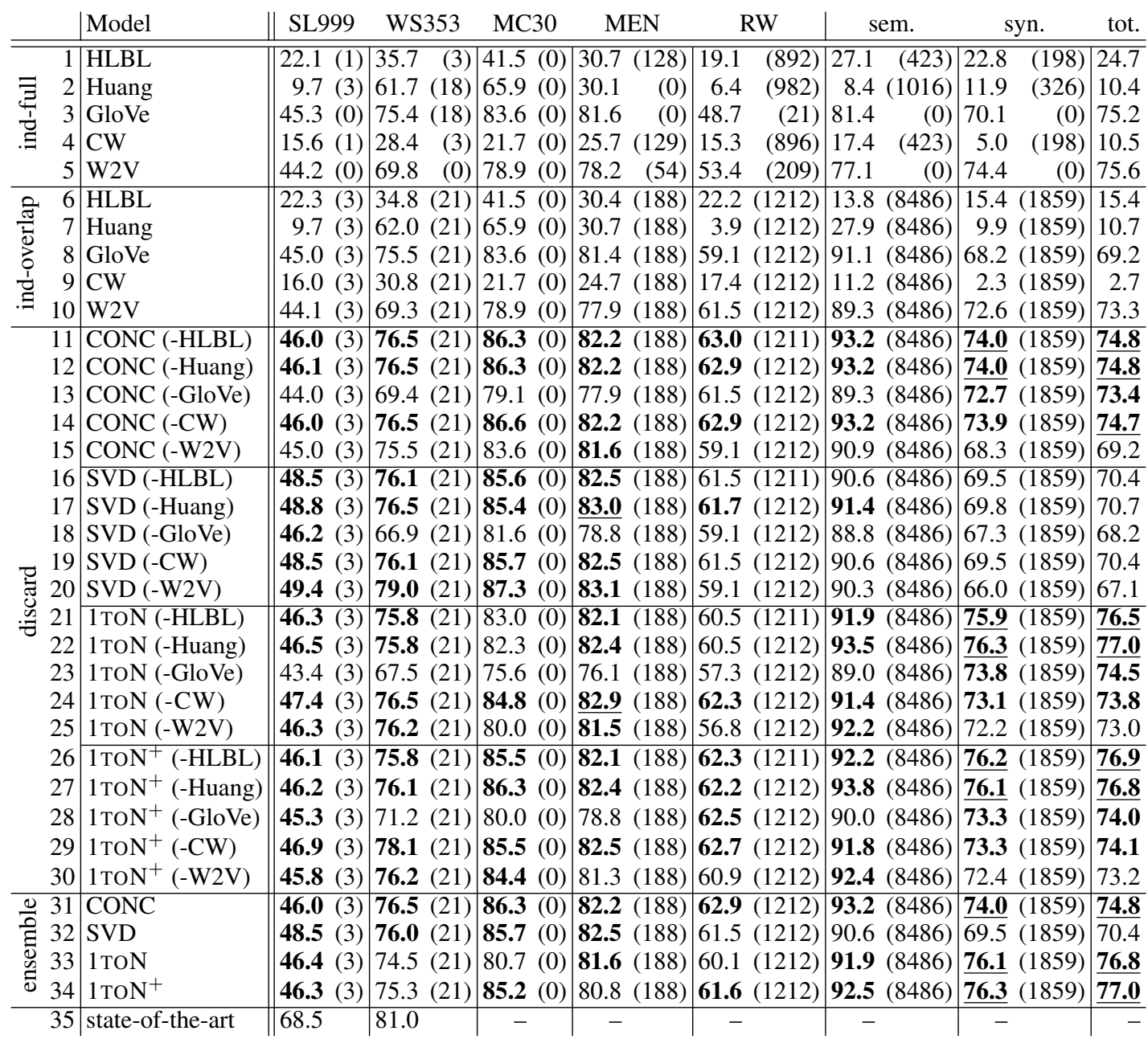

Table 3: Results on five word similarity tasks (Spearman correlation metric) and analogical reasoning (accuracy). The number of OOVs is given in parentheses for each result. "ind-full/ind-overlap": individual embedding sets with respective full/overlapping vocabulary; "ensemble": ensemble results using all five embedding sets; "discard": one of the five embedding sets is removed. If a result is better than all methods in "ind-overlap", then it is bolded. Significant improvement over the best baseline in "ind-overlap" is underlined (online toolkit from http: / /vassarstats. net/index. html for Spearman correlation metric, test of equal proportions for accuracy, $\mathrm{p}<.05$ ).

\begin{tabular}{|c|c|c|c|c|c|c|c|c|c|c|c|c|c|c|c|c|}
\hline & \multicolumn{4}{|c|}{ RW(21) } & \multicolumn{4}{|c|}{ semantic } & \multicolumn{4}{|c|}{ syntactic } & \multicolumn{4}{|c|}{ total } \\
\hline & RND & AVG & $\mathrm{ml}$ & $1 \mathrm{TON}^{+}$ & RND & AVG & $\mathrm{ml}$ & $1 \mathrm{TON}^{+}$ & RND & AVG & $\mathrm{ml}$ & $1 \mathrm{TON}^{+}$ & RND & AVG & $\mathrm{ml}$ & $1 \mathrm{TON}^{+}$ \\
\hline HLBL & 7.4 & 6.9 & 17.3 & 17.5 & 26.3 & 26.4 & 26.3 & 26.4 & 22.4 & 22.4 & 22.7 & 22.9 & 24.1 & 24.2 & 24.4 & 24.9 \\
\hline . Huang & 4.4 & 4.3 & 6.4 & 6.4 & 1.2 & 2.7 & 21.8 & 22.0 & 7.7 & 4.1 & 10.9 & 11.4 & 4.8 & 3.3 & 15.8 & 16. \\
\hline $\mathrm{CW}$ & 7.1 & 10.6 & 17.3 & 17.7 & 17.2 & 17.2 & 16.7 & 18.4 & 4.9 & 5.0 & 5.0 & 5.5 & 10.5 & 10.5 & 10.3 & 11. \\
\hline$\cong \mathrm{CONC}$ & 14.2 & 16.5 & 48.3 & - & 4.6 & 18.0 & 88.1 & - & 62.4 & 15.1 & 74.9 & - & 36.2 & 16.3 & 81.0 & \\
\hline ह્ SVD & 12.4 & 15.7 & 47.9 & - & 4.1 & 17.5 & 87.3 & - & 54.3 & 13.6 & 70.1 & - & 31.5 & 15.4 & 77.9 & \\
\hline D $1 \mathrm{TON}$ & 16.7 & 11.7 & 48.5 & - & 4.2 & 17.6 & 88.2 & - & 60.0 & 15.0 & 76.8 & - & 34.7 & 16.1 & 82.0 & \\
\hline ฮี $1 \mathrm{TON}^{+}$ & - & - & - & 48.8 & - & - & - & 88.4 & - & - & - & 76.3 & - & - & - & 81. \\
\hline
\end{tabular}

Table 4: Comparison of effectiveness of four methods for learning OOV embeddings. RND: random initialization. AVG: average of embeddings of known words. ml: MUtUALLEARNING. RW(21) means there are still 21 OOVs for the vocabulary union. 


\subsection{Word Similarity and Analogy Tasks}

We evaluate on SimLex-999 (Hill et al., 2015b), WordSim353 (Finkelstein et al., 2001), MEN (Bruni et al., 2014) and RW (Luong et al., 2013). For completeness, we also show results for MC30, the validation set.

The word analogy task proposed in (Mikolov et al., 2013b) consists of questions like, " $a$ is to $b$ as $c$ is to _ ?". The dataset contains 19,544 such questions, divided into a semantic subset of size 8869 and a syntactic subset of size 10,675 . Accuracy is reported.

We also collect the state-of-the-art report for each task. SimLex-999: (Wieting et al., 2015), WS353: (Halawi et al., 2012). Not all state-ofthe-art results are included in Table 3. One reason is that a fair comparison is only possible on the shared vocabulary, so methods without released embeddings cannot be included. In addition, some prior systems can possibly generate better performance, but those literature reported lower results than ours because different hyperparameter setup, such as smaller dimensionality of word embeddings or different evaluation metric. In any case, our main contribution is to present ensemble frameworks which show that a combination of complementary embedding sets produces betterperforming metaembeddings.

Table 3 reports results on similarity and analogy. Numbers in parentheses are the sizes of words in the datasets that are uncovered by intersection vocabulary. We do not consider them for fair comparison. Block "ind-full" (1-5) lists the performance of individual embedding sets on the full vocabulary. Results on lines 6-34 are for the intersection vocabulary of the five embedding sets: "ind-overlap" contains the performance of individual embedding sets, "ensemble" the performance of our four ensemble methods and "discard" the performance when one component set is removed.

The four ensemble approaches are very promising (31-34). For CONC, discarding HLBL, Huang or $\mathrm{CW}$ does not hurt performance: CONC (31), CONC(-HLBL) (11), CONC(-Huang) (12) and $\mathrm{CONC}(-\mathrm{CW})$ (14) beat each individual embedding set (6-10) in all tasks. GloVe contributes most in SimLex-999, WS353, MC30 and MEN; word2vec contributes most in RW and word analogy tasks.

SVD (32) reduces the dimensionality of CONC from 950 to 200, but still gains performance in SimLex-999 and MEN. GloVe contributes most in
SVD (larger losses on line 18 vs. lines 16-17, 1920). Other embeddings contribute inconsistently.

1 TON performs well only on word analogy, but it gains great improvement when discarding $\mathrm{CW}$ embeddings (24). $1 \mathrm{TON}^{+}$performs better than $1 \mathrm{TON}$ : it has stronger results when considering all embedding sets, and can still outperform individual embedding sets while discarding HLBL (26), Huang (27) or CW (29).

These results demonstrate that ensemble methods using multiple embedding sets produce stronger embeddings. However, it does not mean the more embedding sets the better. Whether an embedding set helps, depends on the complementarity of the sets and on the task.

CONC, the simplest ensemble, has robust performance. However, size-950 embeddings as input means a lot of parameters to tune for DNNs. The other three methods (SVD, 1TON, $1 \mathrm{TON}^{+}$) have the advantage of smaller dimensionality. SVD reduces CONC's dimensionality dramatically and still is competitive, especially on word similarity. 1TON is competitive on analogy, but weak on word similarity. $1 \mathrm{TON}^{+}$performs consistently strongly on word similarity and analogy.

Table 3 uses the metaembeddings of intersection vocabulary, hence it shows directly the quality enhancement by our ensemble approaches; this enhancement is not due to bigger coverage.

System comparison of learning $\mathrm{OOV}$ embeddings. In Table 4, we extend the vocabularies of each individual embedding set ("ind" block) and our ensemble approaches ("ensemble" block) to the vocabulary union, reporting results on RW and analogy - these tasks contain the most OOVs. As both word2vec and GloVe have full coverage on analogy, we do not rereport them in this table. This subtask is specific to "coverage" property. Apparently, our mutual learning and $1 \mathrm{TON}^{+}$can cover the union vocabulary, which is bigger than each individual embedding sets. But the more important issue is that we should keep or even improve the embedding quality, compared with their original embeddings in certain component sets.

For each embedding set, we can compute the representation of an OOV (i) as a randomly initialized vector (RND); (ii) as the average of embeddings of all known words (AVG); (iii) by MUTUALLEARNING (ml) and (iv) by $1 \mathrm{TON}^{+} \cdot 1 \mathrm{TON}^{+}$ learns OOV embeddings for individual embedding sets and metaembeddings simultaneously, and it 


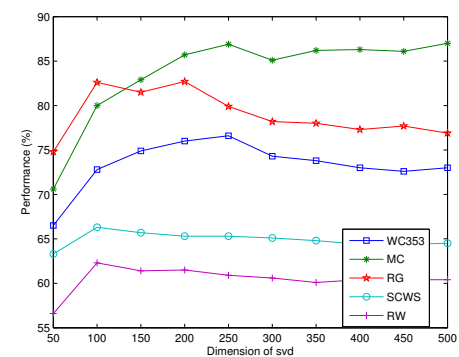

(a) Performance vs. $d$ of SVD

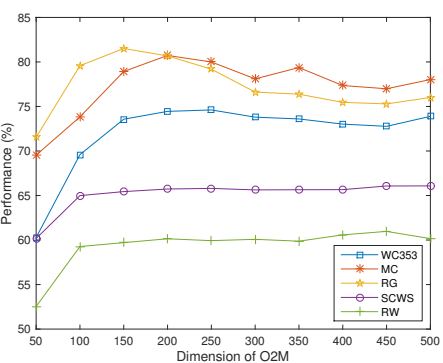

(b) Performance vs. $d$ of $1 \mathrm{TON}$

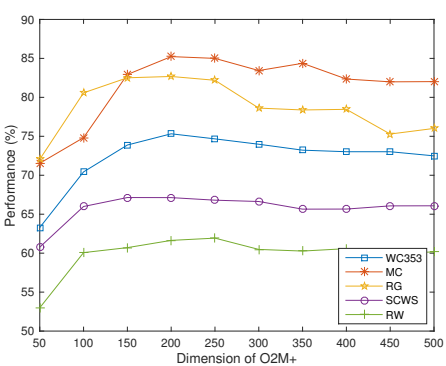

(c) Performance vs. $d$ of $1 \mathrm{TON}^{+}$

Figure 4: Influence of dimensionality

\begin{tabular}{|c|c|c|c|c|c|c|c|c|c|c|}
\hline & newsgroups & revi & ews & web & $\log s$ & ansv & vers & em & ails & $\begin{array}{c}\text { wsj } \\
\text { ALL } \\
\text { OOV }\end{array}$ \\
\hline TnT & 88.6654 .73 & 90.40 & 56.75 & 93.33 & 74.17 & 88.55 & 48.32 & 88.14 & 58.09 & 95.7688 .30 \\
\hline @ Stanford & 89.1156 .02 & 91.43 & 58.66 & 94.15 & 77.13 & 88.92 & 49.30 & 88.68 & 58.42 & 96.8390 .25 \\
\hline SVMTool & 89.1453 .82 & 91.30 & 54.20 & 94.21 & 76.44 & 88.96 & 47.25 & 88.64 & 56.37 & 96.6387 .96 \\
\hline है C\&P & 89.5157 .23 & 91.58 & 59.67 & 94.41 & 78.46 & 89.08 & 48.46 & 88.74 & 58.62 & 96.7888 .65 \\
\hline FLORS & 90.8666 .42 & 92.95 & 75.29 & 94.71 & 83.64 & 90.30 & 62.15 & 89.44 & 62.61 & 96.5990 .37 \\
\hline FLORS+HLBL & 90.0162 .64 & 92.54 & 74.19 & 94.19 & 79.55 & 90.25 & 62.06 & 89.33 & 62.32 & 96.5391 .03 \\
\hline$\geq$ FLORS+Huang & 90.6868 .53 & 92.86 & 77.88 & 94.71 & 84.66 & 90.62 & 65.04 & 89.62 & 64.46 & 96.6591 .69 \\
\hline FLORS+GloVe & 90.9970 .64 & 92.84 & 78.19 & 94.69 & 86.16 & 90.54 & 65.16 & 89.75 & 65.61 & 96.6592 .03 \\
\hline${ }^{\mp} \mathrm{FLORS}+\mathrm{CW}$ & 90.3769 .31 & 92.56 & 77.65 & 94.62 & 84.82 & 90.23 & 64.97 & 89.32 & 65.75 & 96.5891 .36 \\
\hline FLORS+W2V & 90.7272 .74 & 92.50 & 77.65 & 94.75 & 86.69 & 90.26 & 64.91 & 89.19 & 63.75 & 96.4091 .03 \\
\hline FLORS+CONC & 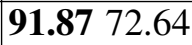 & 92.92 & $\begin{array}{l}78.34 \\
\end{array}$ & 95.37 & 86.69 & 90.69 & 65.77 & 89.94 & 66.90 & 97.3192 .69 \\
\hline 锑 FLORS+SVD & 90.9870 .94 & 92.47 & 77.88 & 94.50 & 86.49 & 90.75 & 64.85 & 89.88 & 65.99 & 96.4290 .36 \\
\hline$\nsubseteq$ FLORS+1TON & 91.5372 .84 & 93.58 & 78.19 & 95.65 & 87.62 & 91.36 & 65.36 & 90.31 & 66.48 & 97.66 92.86 \\
\hline $\mathrm{FLORS}+1 \mathrm{TON}^{+}$ & $\mathbf{9 1 . 5 2} 72.34$ & 93.14 & 78.32 & 95.65 & 87.29 & 90.77 & 65.28 & 89.93 & 66.72 & 97.14 92.55 \\
\hline
\end{tabular}

Table 5: POS tagging results on six target domains. "baselines" lists representative systems for this task, including FLORS. "+indiv / +meta": FLORS with individual embedding set / metaembeddings. Bold means higher than "baselines" and "+indiv".

would not make sense to replace these OOV embeddings computed by $1 \mathrm{TON}^{+}$with embeddings computed by "RND/AVG/ml". Hence, we do not report "RND/AVG/ml" results for $1 \mathrm{TON}^{+}$.

Table 4 shows four interesting aspects. (i) MUTUALLEARNING helps much if an embedding set has lots of OOVs in certain task; e.g., MUTUALLEARNING is much better than AVG and RND on RW, and outperforms RND considerably for CONC, SVD and 1 TON on analogy. However, it cannot make big difference for HLBL/CW on analogy, probably because these two embedding sets have much fewer OOVs, in which case AVG and RND work well enough. (ii) AVG produces bad results for CONC, SVD and 1 TON on analogy, especially in the syntactic subtask. We notice that those systems have large numbers of OOVs in word analogy task. If for analogy " $a$ is to $b$ as $c$ is to $d "$, all four of $a, b, c, d$ are OOVs, then they are represented with the same average vector. Hence, similarity between $b-a+c$ and each OOV is 1.0 . In this case, it is almost impossible to predict the correct answer $d$. Unfortunately, methods CONC, SVD and 1 TON have many OOVs, resulting in the low numbers in Table 4. (iii) MUtUaLLEARNING learns very effective embeddings for OOVs. CONC-ml, 1TON-ml and SVD-ml all get better results than word2vec and GloVe on analogy (e.g., for semantic analogy: 88.1, 87.3, 88.2 vs. 81.4 for GloVe). Considering further their bigger vocabulary, these ensemble methods are very strong representation learning algorithms. (iv) The performance of $1 \mathrm{TON}^{+}$for learning embeddings for OOVs is competitive with Mutuallearning. For HLBL/Huang/CW, $1 \mathrm{TON}^{+}$performs slightly better than MUTUALLEARNING in all four met- 
rics. Comparing $1 \mathrm{TON}-\mathrm{ml}$ with $1 \mathrm{TON}^{+}, 1 \mathrm{TON}^{+}$ is better than "ml" on RW and semantic task, while performing worse on syntactic task.

Figure 4 shows the influence of dimensionality $d$ for SVD, 1TON and $1 \mathrm{TON}^{+}$. Peak performance for different data sets and methods is reached for $d \in[100,500]$. There are no big differences in the averages across data sets and methods for high enough $d$, roughly in the interval $[150,500]$. In summary, as long as $d$ is chosen to be large enough (e.g., $\geq 150$ ), performance is robust.

\subsection{Domain Adaptation for POS Tagging}

In this section, we test the quality of those individual embedding embedding sets and our metaembeddings in a Part-of-Speech (POS) tagging task. For POS tagging, we add word embeddings into FLORS $^{7}$ (Schnabel and Schütze, 2014) which is the state-of-the-art POS tagger for unsupervised domain adaptation.

FLORS tagger. It treats POS tagging as a window-based (as opposed to sequence classification), multilabel classification problem using LIBLINEAR, ${ }^{8}$ a linear SVM. A word's representation consists of four feature vectors: one each for its suffix, its shape and its left and right distributional neighbors. Suffix and shape features are standard features used in the literature; our use of them in FLORS is exactly as described in (Schnabel and Schütze, 2014).

Let $f(w)$ be the concatenation of the two distributional and suffix and shape vectors of word $w$. Then FLORS represents token $v_{i}$ as follows:

$f\left(v_{i-2}\right) \oplus f\left(v_{i-1}\right) \oplus f\left(v_{i}\right) \oplus f\left(v_{i+1}\right) \oplus f\left(v_{i+2}\right)$ where $\oplus$ is vector concatenation. Thus, token $v_{i}$ is tagged based on a 5-word window.

FLORS is trained on sections 2-21 of Wall Street Journal (WSJ) and evaluate on the development sets of six different target domains: five SANCL (Petrov and McDonald, 2012) domains newsgroups, weblogs, reviews, answers, emails and sections 22-23 of WSJ for in-domain testing.

Original FLORS mainly depends on distributional features. We insert word's embedding as the fifth feature vector. All embedding sets (except for $1 \mathrm{TON}^{+}$) are extended to the union vocabulary by MutualLearning. We test if this additional feature can help this task.

Table 5 gives results for some representa-

\footnotetext{
${ }^{7}$ Cistern.cis.lmu.de/flors (Yin et al., 2015)

${ }^{8}$ liblinear.bwaldvogel. de (Fan et al., 2008)
}

tive systems ("baselines"), FLORS with individual embedding sets ("+indiv") and FLORS with metaembeddings ("+meta"). Following conclusions can be drawn. (i) Not all individual embedding sets are beneficial in this task; e.g., HLBL embeddings make FLORS perform worse in 11 out of 12 cases. (ii) However, in most cases, embeddings improve system performance, which is consistent with prior work on using embeddings for this type of task (Xiao and Guo, 2013; Yang and Eisenstein, 2014; Tsuboi, 2014). (iii) Metaembeddings generally help more than the individual embedding sets, except for SVD (which only performs better in 3 out of 12 cases).

\section{Conclusion}

This work presented four ensemble methods for learning metaembeddings from multiple embedding sets: CONC, SVD, $1 \mathrm{TON}$ and $1 \mathrm{TON}^{+}$. Experiments on word similarity and analogy and POS tagging show the high quality of the metaembeddings; e.g., they outperform GloVe and word $2 \mathrm{vec}$ on analogy. The ensemble methods have the added advantage of increasing vocabulary coverage. We make our metaembeddings available at http://cistern. cis. lmu.de/meta-emb.

\section{Acknowledgments}

We gratefully acknowledge the support of Deutsche Forschungsgemeinschaft (DFG): grant SCHU 2246/8-2.

\section{References}

Mohit Bansal, Kevin Gimpel, and Karen Livescu. 2014. Tailoring continuous word representations for dependency parsing. In Proceedings of ACL, pages 809-815.

Yoshua Bengio, Réjean Ducharme, Pascal Vincent, and Christian Janvin. 2003. A neural probabilistic language model. JMLR, 3:1137-1155.

Elia Bruni, Nam-Khanh Tran, and Marco Baroni. 2014. Multimodal distributional semantics. JAIR, 49(1-47).

Yanqing Chen, Bryan Perozzi, Rami Al-Rfou, and Steven Skiena. 2013. The expressive power of word embeddings. In ICML Workshop on Deep Learning for Audio, Speech, and Language Processing.

Ronan Collobert and Jason Weston. 2008. A unified architecture for natural language processing: Deep neural networks with multitask learning. In Proceedings of ICML, pages 160-167. 
John Duchi, Elad Hazan, and Yoram Singer. 2011. Adaptive subgradient methods for online learning and stochastic optimization. JMLR, 12:2121-2159.

Rong-En Fan, Kai-Wei Chang, Cho-Jui Hsieh, XiangRui Wang, and Chih-Jen Lin. 2008. LIBLINEAR: A library for large linear classification. $J M L R$, 9:1871-1874.

Lev Finkelstein, Evgeniy Gabrilovich, Yossi Matias, Ehud Rivlin, Zach Solan, Gadi Wolfman, and Eytan Ruppin. 2001. Placing search in context: The concept revisited. In Proceedings of $W W W$, pages 406-414.

Guy Halawi, Gideon Dror, Evgeniy Gabrilovich, and Yehuda Koren. 2012. Large-scale learning of word relatedness with constraints. In Proceedings of $K D D$, pages 1406-1414.

Felix Hill, KyungHyun Cho, Sebastien Jean, Coline Devin, and Yoshua Bengio. 2014. Not all neural embeddings are born equal. In NIPS Workshop on Learning Semantics.

Felix Hill, Kyunghyun Cho, Sebastien Jean, Coline Devin, and Yoshua Bengio. 2015a. Embedding word similarity with neural machine translation. In Proceedings of ICLR Workshop.

Felix Hill, Roi Reichart, and Anna Korhonen. 2015b. Simlex-999: Evaluating semantic models with (genuine) similarity estimation. Computational Linguistics, pages 665-695.

Eric H Huang, Richard Socher, Christopher D Manning, and Andrew Y Ng. 2012. Improving word representations via global context and multiple word prototypes. In Proceedings of ACL, pages 873-882.

Quoc V Le and Tomas Mikolov. 2014. Distributed representations of sentences and documents. In Proceedings of ICML, pages 1188-1196.

Yong Luo, Jian Tang, Jun Yan, Chao Xu, and Zheng Chen. 2014. Pre-trained multi-view word embedding using two-side neural network. In Proceedings of AAAI, pages 1982-1988.

Minh-Thang Luong, Richard Socher, and Christopher D Manning. 2013. Better word representations with recursive neural networks for morphology. In Proceedings of CoNLL, volume 104, pages 104-113.

Tomas Mikolov, Kai Chen, Greg Corrado, and Jeffrey Dean. 2013a. Efficient estimation of word representations in vector space. In Proceedings of ICLR Workshop.

Tomas Mikolov, Ilya Sutskever, Kai Chen, Greg S Corrado, and Jeff Dean. 2013b. Distributed representations of words and phrases and their compositionality. In Proceedings of NIPS, pages 3111-3119.
George A Miller and Walter G Charles. 1991. Contextual correlates of semantic similarity. Language and cognitive processes, 6(1):1-28.

Andriy Mnih and Geoffrey E Hinton. 2009. A scalable hierarchical distributed language model. In Proceedings of NIPS, pages 1081-1088.

Jeffrey Pennington, Richard Socher, and Christopher D Manning. 2014. Glove: Global vectors for word representation. Proceedings of EMNLP, 12:15321543.

Slav Petrov and Ryan McDonald. 2012. Overview of the 2012 shared task on parsing the web. In Proceedings of SANCL, volume 59.

Pushpendre Rastogi, Benjamin Van Durme, and Raman Arora. 2015. Multiview LSA: Representation learning via generalized CCA. In Proceedings of NAACL, pages 556-566.

Tobias Schnabel and Hinrich Schütze. 2014. FLORS: Fast and simple domain adaptation for part-ofspeech tagging. TACL, 2:15-26.

Ilya Sutskever, Oriol Vinyals, and Quoc VV Le. 2014. Sequence to sequence learning with neural networks. In Proceedings of NIPS, pages 3104-3112.

Yuta Tsuboi. 2014. Neural networks leverage corpuswide information for part-of-speech tagging. In Proceedings of EMNLP, pages 938-950.

Joseph Turian, Lev Ratinov, and Yoshua Bengio. 2010. Word representations: a simple and general method for semi-supervised learning. In Proceedings of ACL, pages 384-394.

John Wieting, Mohit Bansal, Kevin Gimpel, and Karen Livescu. 2015. From paraphrase database to compositional paraphrase model and back. TACL, $3: 345-358$.

Min Xiao and Yuhong Guo. 2013. Domain adaptation for sequence labeling tasks with a probabilistic language adaptation model. In Proceedings of ICML, pages 293-301.

Yi Yang and Jacob Eisenstein. 2014. Unsupervised domain adaptation with feature embeddings. In Proceedings of ICLR Workshop.

Wenpeng Yin and Hinrich Schütze. 2015. Multichannel variable-size convolution for sentence classification. In Proceedings of CoNLL, pages 204-214.

Wenpeng Yin, Tobias Schnabel, and Hinrich Schütze. 2015. Online updating of word representations for part-of-speech taggging. In Proceedings of EMNLP, pages 1329-1334.

Ye Zhang, Stephen Roller, and Byron Wallace. 2016. MGNC-CNN: A simple approach to exploiting multiple word embeddings for sentence classification. In Proceedings of NAACL-HLT. 